\title{
A Blind Survey of the Local Dusty Universe with Herschel-ATLAS
}

Christopher Clark*

Cardiff University

E-mail: Christopher.Clarkdastro.cf.ac.uk

\section{Haley Gomez}

Cardiff University

\section{Loretta Dunne}

University Of Canterbury

\section{Pieter de Vis}

University Of Canterbury

\section{Steve Maddox}

University Of Canterbury

\begin{abstract}
A blind survey of the nearby dusty galaxies detected by Herschel-ATLAS reveals that $75 \%$ are, unexpectedly, irregular and/or highly flocculent in morphology. They also tend to exhibit extremely blue UV-NIR colour; these galaxies are bluer than $90 \%$ of galaxies seen in targeted dust surveys. They are also colder, with a median dust temperature of only $17 \mathrm{~K}$, and are 3 times dustier. Most have greater gas mass than stellar mass, with a median atomic gas fraction of 51\%, with values ranging as high as $99 \%$.
\end{abstract}

The Life Cycle of Dust in the Universe: Observations, Theory, and Laboratory Experiments 18-22 November, 2013

Taipei, Taiwan

\footnotetext{
* Speaker.
} 

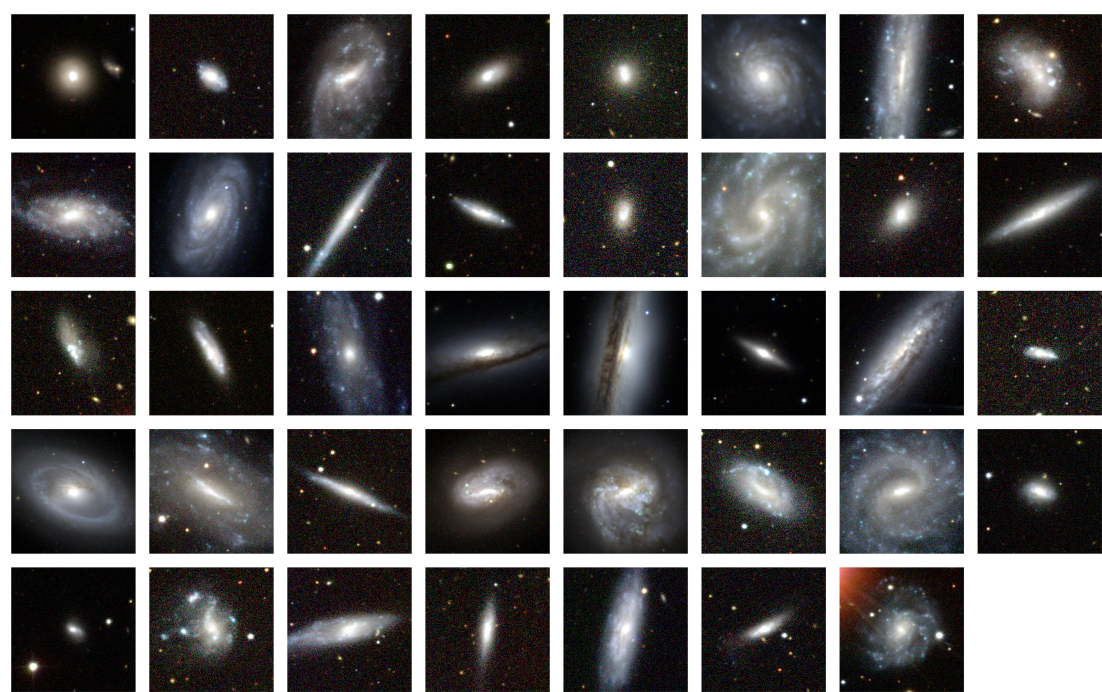

Figure 1: Optical SDSS gri-band three-colour imagery of the HAPLESS galaxies. Each thumbnail is 100" on a side. Note the blue colours, and preponderance of irregular and flocculent morphologies.

\section{Introduction and The HAPLESS Sample}

The Herschel-ATLAS[W] is a blind survey of 550 square degrees of sky, at five far-infrared (FIR) wavelengths between 100-500 $\mu \mathrm{m}$. H-ATLAS gives us the best of both worlds: a blind and unbiased coverage of a large area of sky, but with resolution and sensitivity previously only found in small-area, targeted FIR surveys.

Using the $H$-ATLAS internal phase-1 data release (162 square degrees), we created a local, volume-limited sample of all 39 galaxies detected by Herschel[[]] in a distance range of 15-45 Mpc. We named this sample HAPLESS: the Herschel-Atlas Phase-1 Limited Extent Spatial Survey. The HAPLESS galaxies are shown in Figure $\square$ as they appear in the optical (Clark et al., in prep).

\section{Peculiar Blue Galaxies}

A surprisingly large number of the HAPLESS galaxies exhibit "atypical" morphologies, with classifications from EFIGI [B]. We find that $54 \%$ of our blind sample are irregular (Hubble type T >8), compared to $25 \%$ in targeted surveys such as KINGFISH[B] and the Herschel Reference Survey[四] (HRS, see Section [1]). Furthermore, many of the HAPLESS galaxies exhibit a high degree of flocculence. In total, $75 \%$ were designated by EFIGI to be either irregular, highly flocculent, or both.

A majority of the HAPLESS galaxies have extremely blue UV-NIR (ultraviolet minus nearinfrared) colour. These very blue galaxies tend to be the ones that have non-standard morphologies. We find a colour criterion of FUV- $K_{s}<3.5$ reliably identifies these very blue interesting objects. The submm and UV brightness of these galaxies indicates large dust masses and high rates of star formation, whilst NIR faintness suggests a relatively modest evolved stellar population. Multiwavelength imagery of four of the these galaxies can be found in Figure $\square$. 

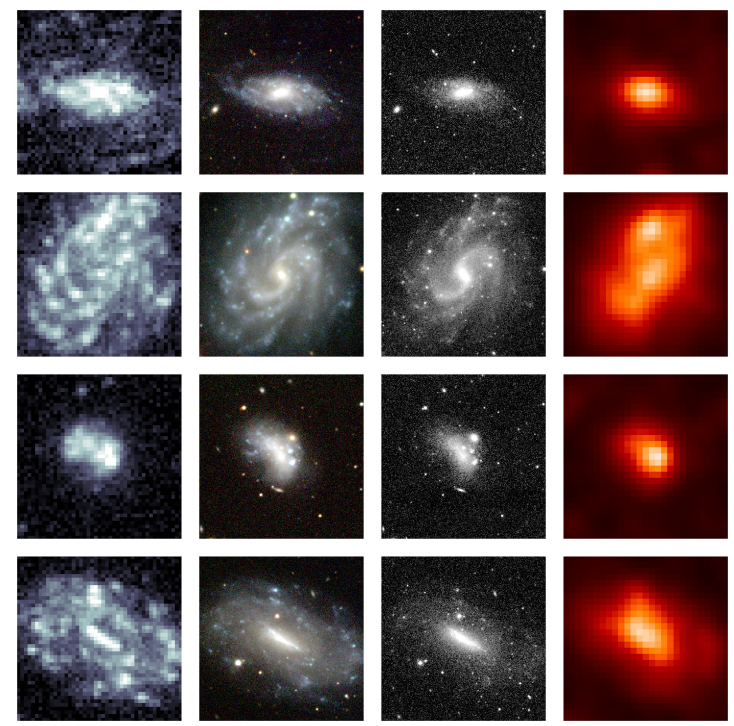

Figure 2: Multiwavelength imagery of four examples of the UV- and submm-luminous, very blue, flocculent galaxies found in the HAPLESS sample. The bands displayed, from top-to-bottom, are: GALEX FUV, SDSS gri-band three-colour, VIKING $K_{S}$-band, and PSF-filtered Herschel $250 \mu \mathrm{m}$. Each image is 150" on a side. Note the blue optical colours, flocculent morphologies, NIR faintness, and bright extended UV emission.

\section{Comparisons with a Targeted Survey}

In Figure [1, we compare the HAPLESS galaxies to the 323 galaxies of the HRS. the largest targeted FIR nearby galaxy survey, a volume-limited sample selected by $K_{s}$-band apparent magnitude. To ensure a valid direct comparison, we use the same spectral energy distribution (SED) fitting procedure on both samples, where we attempt $\chi^{2}$-minimising fits with both one- and two-component modified blackbody models.

The upper left plot of Figure B 3 shows that the dust in an average HAPLESS galaxy is colder (with median $\mathrm{T}_{\text {dust }}=17 \mathrm{~K}$ ) than the dust in $80 \%$ of the galaxies of the HRS, and bluer than $90 \%$; the HRS undersampled the cold blue galaxies seen in a blind survey. This is reinforced by the upper right plot, which demonstrates the different cold dust temperature distributions of the two samples, showing the prevalence of galaxies with $\mathrm{T}_{\text {dust }}<15 \mathrm{~K}$ in a blind sample.

In the lower left plot of Figure B, the HAPLESS galaxies are shown to have dust masses which are on average 3 times greater than the HRS for a given stellar mass. Whilst it is unsurprising that a sample selected by dust emission will generally contain more dust-rich objects than a sample selected by stellar emission, the nature of this dusty population is particularly interesting.

The HAPLESS galaxies, particularly those with FUV- $K_{s}<3.5$, exhibit high gas fractions, which are plotted against FUV- $K_{s}$ in the lower right plot of Figure B (HI masses derived from

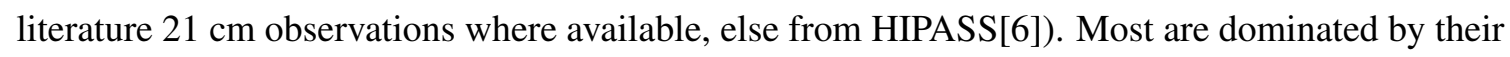
gas component (median gas fraction 51\%), with gas fractions as high as 94 and $99 \%$. The majority of the gas-rich HAPLESS galaxies have FUV- $K_{S}<3.5$, suggesting that these are objects that have simply converted less of their gas into stars. 

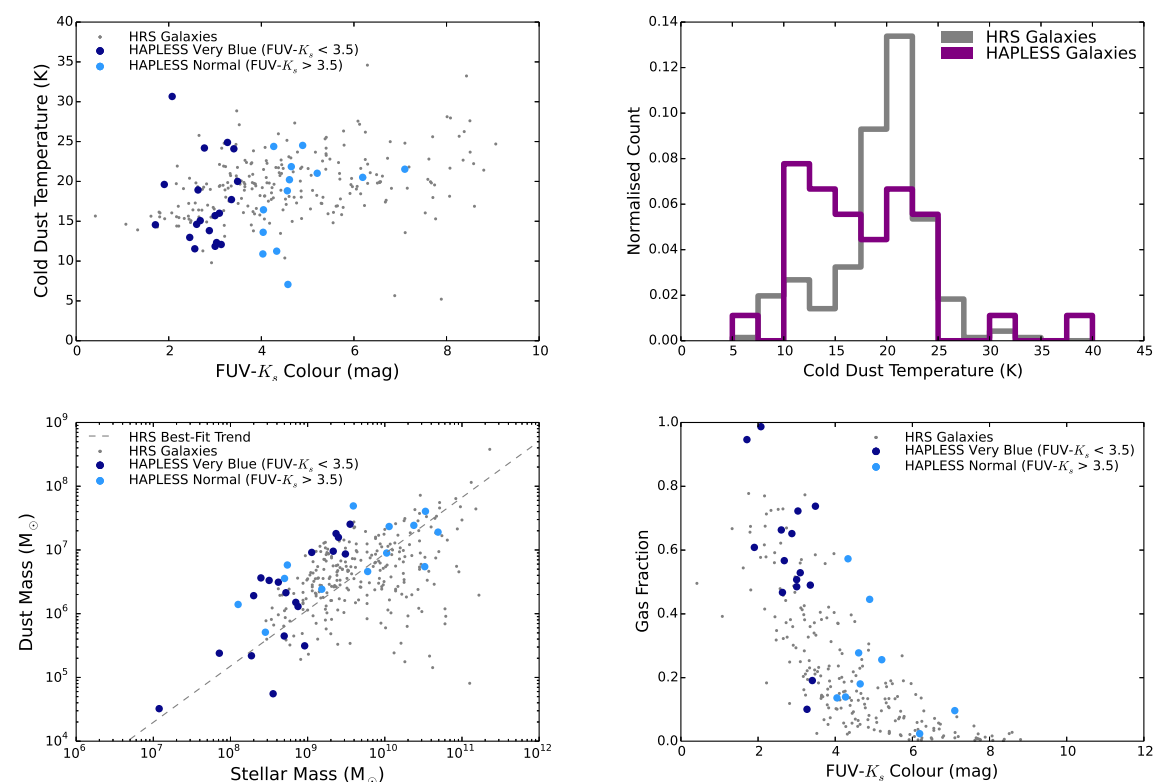

Figure 3: Plots comparing our blind HAPLESS sample with the targeted HRS sample. HAPLESS galaxies are marked in colour; in the scatter plots, point colour indicates whether a galaxy is bluer than the FUV- $K_{s}$ $<3.5$ colour criterion. The HRS galaxies are marked in grey. Upper left: Plot of FUV- $K_{s}$ colour against cold dust temperature. Upper right: Histogram of cold dust temperatures. Lower left: Plot of stellar mass against dust mass. Lower right: Plot of FUV- $K_{s}$ colour against (atomic) gas fraction.

\section{Conclusion}

HAPLESS, the first submm-selected sample of nearby galaxies, reveals that cold dust temperatures and high gas fractions appear to be the norm amongst dusty galaxies - a fact missed by targeted surveys. Along with their very blue UV-NIR colours, this suggests that these are younger systems that have nonetheless processed a lot of their gas into dust. This should allow us to use these galaxies to probe the origins of dust, and put constraints upon chemical evolution.

\section{References}

[1] S. Eales et al, The Herschel ATLAS, PASP (2010) 122499 [arXiv:0 910 . 4279].

[2] G. L. Pilbratt et al, Herschel Space Observatory - An ESA facility for far-infrared and submillimetre astronomy, $A \& A$ (2010) $518 \mathrm{~L} 1$ [arXiv1005.5331]

[3] A. Baillard et al, The EFIGI catalogue of 4458 nearby galaxies with detailed morphology, A\&A (2011) 532 A74 [arXiv:1103.5734].

[4] R. C. Kennicutt, KINGFISH - Key Insights on Nearby Galaxies: A Far-Infrared Survey with Herschel: Survey Description and Image Atlas, PASP (2011) 1231347 [1111 . 4 438]

[5] A. Boselli et al, The Herschel Reference Survey, PASP (2010) 122261 [arXiv: 1001.5136 ].

[6] M. J. Meyer, The HIPASS catalogue - I. Data presentation, MNRAS (2004) 3501195 [arXiv:astro-ph/0406384] 